\title{
Author Correction: Colonic diverticular disease
}

Antonio Tursi, Carmelo Scarpignato, Lisa L. Strate, Angel Lanas, Wolfgang Kruis, Adi Lahat and Silvio Danese

Published: 26 March 2020 Nature Reviews Disease Primers 6, Article number: 20 (2020) https://doi.org/10.1038/s41572-020-0153-5

Published online 26 March 2020

In the originally published version, the affiliation for Silvio Danese was incorrect. His affiliations have now been corrected in both the HTML and PDF versions of the article, to state: IBD Center, Department of Gastroenterology, Humanitas Clinical and Research Center - IRCCS -, Rozzano, Italy, and Department of Biomedical Sciences, Humanitas University, Pieve Emanuele, Italy.

https://doi.org/10.1038/s41572-020-0192-y | Published online: 17 June 2020

(๑) Springer Nature Limited 2020 